\title{
Optimal tuning of a wind plant energy production based on improved grey wolf optimizer
}

\author{
Mohd Zaidi Mohd Tumari', Mohd Muzaffar Zahar², Mohd Ashraf Ahmad \\ ${ }^{1}$ Fakulti Teknologi Kejuruteraan Elektik \& Elektronik, Universiti Teknikal Malaysia Melaka, Malaysia \\ ${ }^{2}$ Department of Electrical Engineering, Centre for Diploma Studies, Universiti Tun Hussein Onn, Malaysia \\ ${ }^{3}$ Faculty of Electrical and Electronics Engineering Technology, University Malaysia Pahang, Malaysia
}

\begin{tabular}{l} 
Article Info \\
\hline Article history: \\
Received Jun 1, 2018 \\
Revised Jul 10, 2018 \\
Accepted Jul 25, 2018 \\
\hline
\end{tabular}

\section{Keywords:}

Energy production Metaheuristic algorithm Nature inspired algorithm Wake interactions Wind farm

\begin{abstract}
The tuning of optimal controller parameters in wind plant is crucial in order to minimize the effect of wake interaction between turbines. The purpose of this paper is to develop an improved grey wolf optimizer (I-GWO) in order to tune the controller parameters of the turbines so that the total energy production of a wind plant is increased. The updating mechanism of original GWO is modified to improve the efficiency of exploration and exploitation phase while avoiding trapping in local minima solution. A row of ten turbines is considered to evaluate the effectiveness of the I-GWO by maximizing the total energy production. The proposed approach is compared with original GWO and previously published modified GWO. Finally, I-GWO produces the highest total energy production as compared to other methods, as shown in statistical performance analysis.
\end{abstract}

This is an open access article under the CC BY-SA license.

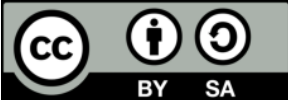

\section{Corresponding Author:}

Mohd Zaidi Mohd Tumari,

Fakulti Teknologi Kejuruteraan Elektik \& Elektronik,

Universiti Teknikal Malaysia Melaka,

Jln Hang Tuah Jaya, 76100, Melaka, Malaysia.

Email: mohdzaidi.tumari@utem.edu.my

\section{INTRODUCTION}

Nowadays, wind plant research has the main issue which is a decrease in total energy production that leads to an increase in price of electricity. This is because of the wake interactions or turbulence between turbines in the wind plant. Thus, it is essential to fine-tune the optimal controller parameters of each turbine in the wind plant. The controller parameters for the turbines are the angle of blade and yaw or/and torque generator. Usually, to find the controller parameters for a single turbine is much simpler by using a model-based controller. Unfortunately, went it comes to an array of turbines, the task in optimizing the controller parameters will be more difficult and complicated. This complexity is because of difficulty in studying the dynamic behavior of the wake interactions between turbines where the turbulence is always happened in chaotic behavior and highly depended on different wind directions and speed magnitude. Therefore, the model-based approach is not suitable in finding optimal controller parameters of the wind plant.

Meanwhile, a model-free or data-driven approach, which is well-known with the design of the controller without knowing an exact model of the system will be a more attractive method to be implemented in the wind plant. A considerable amount of literature has been published on finding the optimal controller parameters of an array of turbines based on the data-driven method that only depends on the total energy production data of wind plant. Multi-resolution simultaneous perturbation stochastic approximation (MR-SPSA) [1], game-theoretic [2-4], gradient-based optimization [5] and optimized relative step size 
random search (ORSSRS) [6] are the examples of data-driven method that focused on single-agent-based optimization. The limitation of single-agent-based optimization is the solution tends to trap in local optima solutions. On the other hand, multi-agent-based optimization performs optimization using a set of solutions (population). Thus, multi-agent-based generally have better exploration compared to single-agent-based, so, better chance to avoid local optima solutions. Recently, a considerable literature has grown up around the theme of multi-agent-based optimization for wind plant. This include, spiral dynamics algorithm [7], particle swarm optimization [7], Bayesian ascent (BA) [8], sine-cosine algorithm [9] and moth flame optimization [10].

Alternatively, a grey wolf optimizer (GWO) [11], which is swarm-based inspired by social behavior of groups of animals (grey wolves), has been successfully solved numerous types of real applications. For instance, improving wind plant production [12], solving optimal reactive power dispatch problem [13], automatic generation control of interconnected power system [14], design for a photovoltaic (PV) [15, 16], vehicle engine [17], unmanned aerial vehicle (UAV) [18], facial image [19], image segmentation [20], gridconnected permanent-magnet synchronous generator [21], satellite image segmentation [22], hybrid renewable energy system PV-diesel generator-battery [23], and liquid slosh system identification [24]. GWO algorithm is inspired by the social hierarchy of grey wolves that divided into four groups, which are alphas, beta, delta and omega. These grey wolves are hunting the prey by following methods: 1) Finding, chasing and reaching prey, 2) Surrounding and harassing prey till it becomes standstill and 3) Attacking the prey. Unfortunately, based on our preliminary works using a row of ten turbines, a major problem with the original GWO is premature convergence which is the solution is not optimum. This problem will lead to low accuracy of total energy production. So far, however, there has been little discussion about the modification of original GWO algorithm for wind plant application. For that reason, this paper is proposed to improve the original GWO algorithm in order to get a better total energy production of a wind plant.

The purpose of this paper is to improve the total energy production of wind plant based on the modification of original GWO, named as improved grey wolf optimizer (I-GWO). The original GWO uses a linear updated mechanism in the algorithm to balance the exploration and exploitation phase. In our modification, we purpose a new nonlinear updated mechanism to provide an optimal portion of exploration and exploitation for wind plant problem. Moreover, the efficacy of the I-GWO is evaluated with a single row wind plant with ten turbines. Here, the wind plant modelling from Park model [25] is considered to verify the effectiveness of the proposed technique. The number of agents and the maximum number of iterations is investigated to find the optimum combination in providing high accuracy of the total energy production. Since the I-GWO is a multi-agent optimization, there will be a huge number of trials to evaluate its consistency in maximizing the total energy production. The performance of the proposed method is analyzed statistically in terms of mean, best, worst and standard deviation of the wind plant total energy production. Furthermore, the results are compared with the previously published modified GWO [12] and the original GWO approaches.

\section{PROBLEM FRAMEWORK}

In this section, the problem framework of the total energy production of the wind plant is explained. The energy production of turbine $k$ can be defined as $E_{k}\left(h_{1}, h_{2}, \ldots, h_{m}\right)(k=1,2, \ldots, m)$, where $h_{k}(k=1,2, \ldots, m)$ is the controller parameter of each turbine $k$ and $m$ is the total number of turbines in the wind plant. Logically, the wind plant received the wind at a different speed and angle of directions with a random position of turbines. Therefore, the energy production $E_{k}$ of turbine $k$ can be influenced by the controller parameters of other turbines $h_{1}, h_{2}, \ldots, h_{k-1}, h_{k+1}, \ldots, h_{m}$, which is not included in controller parameter of turbine $k$. This is due to the wake interactions between turbines. Same goes to change of $h_{k}$ that might influence the energy productions of other turbines $E_{1}, E_{2}, \ldots, E_{k-1}, E_{k+1}, \ldots, E_{m}$. The exact mathematical model of function $E_{k}\left(h_{1}, h_{2}, \ldots, h_{m}\right)$ is difficult to derive due to highly complex turbulence interactions between turbines, and thus, in this study, it is negligible. On the other hand, the data on the energy production of each turbine is measurable. Therefore, the total measured energy production is stated as follows:

$$
\bar{E}\left(h_{1}, h_{2}, \ldots, h_{m}\right)=\sum_{k=1}^{m} E_{k}\left(h_{1}, h_{2}, \ldots, h_{m}\right)
$$

Finally, this wind plant data-driven control problem can be stated as:

Problem 2.1: Find the controller parameter of each turbine $h_{k}(k=1,2, \ldots, m)$ such that the total energy production $\bar{E}\left(h_{1}, h_{2}, \ldots, h_{m}\right)$ in (1) is maximized without any knowledge on the relation between $h_{k}(k=1,2, \ldots$, $m)$ and $\bar{E}$. 


\section{WIND PLANT MATHEMATICAL MODEL}

The mathematical model for wind plant in [25] is briefly explained in this section. The energy production of each turbine $k$ can be considered as:

$$
E_{k}=2 \rho A_{k} h_{k}\left(1-h_{k}\right)^{2} W_{k}
$$

In (2) $\rho, A_{k}$ and $W_{k}$ are the air density, rotor swept area of turbine $k$ and accumulation wind speed produced by the upstream wind turbines, respectively. The equation of $W_{k}$ is defined as:

$$
W_{k}=U\left(1-2 \sqrt{\sum_{l=1}^{F}\left(h_{l}\left(\frac{D_{l}}{D_{l}+2 \phi d_{l k}}\right)^{2} \frac{A_{o v}}{A_{k}}\right)^{2}}\right)
$$

where $U, F, l, D_{l}, \varphi, d_{l k}$ and $A_{o v}$ are the incoming wind speed in front of the first turbine, the number of upstream turbine, the index to represent the upstream turbine, the diameter of turbine rotor, the parameter to represent the gradient of the wind turbulence between two turbines, the distance between turbine $k$ and upstream turbine $l$ and the overlap area of turbulence from the upstream turbine, respectively.

Remarks: Note that in this research, only the data of total energy production of a wind plant $\bar{E}$ is needed without any knowledge of exact relation between $\bar{E}\left(h_{1}, h_{2}, \ldots, h_{m}\right)$ and $h_{k}(k=1,2, \ldots, m)$ as stated in $(2,3)$. Hence, the data-driven or model-free-based control is suitable to implement in real wind plant optimization problem due to it only depends on the total energy production data.

\section{IMPROVED GREY WOLF OPTIMIZER}

The proposed I-GWO for finding the optimal controller parameters of wind turbines in a wind plant is explained in this section. Firstly, an original grey wolf optimizer (GWO) is introduced. Then, the modification in GWO is explained in detail, which can provide better total energy production of the wind plant.

\subsection{Original GWO}

The original GWO algorithm is introduced by [11] that motivated by the grey wolves hunting for prey in nature. Here, a brief explanation of the original GWO is presented. Let $g: \boldsymbol{R}^{n} \rightarrow \boldsymbol{R}$, $\boldsymbol{v}_{i}(i=1,2, \ldots, N)$ and $N$ are the cost function, design variable and the number of agents, respectively. And, let $v_{i j}(j=1,2, \ldots, n)$ is $j$-th element of the vector $\boldsymbol{v}_{i}$. Therefore, a maximization problem can be expressed as:

$$
\arg \max _{\mathbf{v}_{i}(1), \mathbf{v}_{i}(2), . .} g_{i}\left(\mathbf{v}_{i}(t)\right)
$$

for iteration $t=1,2, \ldots$ and for each agent $i$. Then, the design variable vector for each agent is updated iteratively as:

$$
\mathbf{v}_{i}(t+1)=\frac{\mathbf{v}^{1}+\mathbf{v}^{2}+\mathbf{v}^{3}}{3}
$$

where:

$$
\mathbf{v}^{1}=\mathbf{v}^{\alpha}-\mathbf{A} \cdot\left|\mathbf{C} \cdot \mathbf{v}^{\alpha}-\mathbf{v}_{i}(t)\right|, \mathbf{v}^{2}=\mathbf{v}^{\beta}-\mathbf{A} \cdot\left|\mathbf{C} \cdot \mathbf{v}^{\beta}-\mathbf{v}_{i}(t)\right|, \mathbf{v}^{3}=\mathbf{v}^{\delta}-\mathbf{A} \cdot\left|\mathbf{C} \cdot \mathbf{v}^{\delta}-\mathbf{v}_{i}(t)\right|
$$

for $i=1,2, \ldots, N$, where the vectors $\boldsymbol{A}$ and $\boldsymbol{C}$ are formulated as follows:

$$
\begin{aligned}
& \mathbf{A}=2 \mathbf{a} \cdot \mathbf{r}_{1}-\mathbf{a}, \\
& \mathbf{C}=2 \cdot \mathbf{r}_{2},
\end{aligned}
$$

where $\boldsymbol{r}_{1}$ and $\boldsymbol{r}_{2}$ are pseudorandom numbers in the range [0,1] and components of $\boldsymbol{a}$ is linearly decreased from 2 to 0 over the course of iteration using the following equation: 


$$
a=2\left(1-\frac{t}{T}\right)
$$

where $T$ is the maximum number of iterations. Note that the random vectors $\boldsymbol{r}_{1}$ and $\boldsymbol{r}_{2}$ of $\boldsymbol{A}$ and $\boldsymbol{C}$ in (6), are generated independently between $v^{1}, v^{2}$ and $v^{3}$. Moreover, the optimal solution is based on the hierarchy of the wolves, which is a priority is from $v^{\alpha}$, followed by $v^{\beta}$ and $v^{\delta}$.

\subsection{Improved GWO}

In this section, the modification of the original GWO algorithm is explained. As stated in (9), the value of $a$ of original GWO is linearly decreased from 2 to 0 over the course of an iteration. The linearity of the original updating mechanism is claimed to provide an exact balance between exploration and exploitation phase. However, in (9) there is no parameter to adjust in order to vary the percentage of exploration and exploitation. Thus, this setting is only limited to several applications. Therefore, a nonlinear equation for updating mechanism $a$ with parameters that can be adjusted seems to be a good choice to cater to this problem. A modified updating mechanism of GWO to produce a nonlinear equation of updated step size is shown as follows:

$$
\tilde{a}=\sigma(\tanh (\mu(t-T)))^{\lambda}
$$

In (10), the symbols $\sigma, \mu$ and $\lambda$ are the positive constant values that are introduced to regulate the portion of exploration and exploitation during the tuning process. The proposed nonlinear updating mechanism for different values of $\mu, \sigma$ equal to 2 and $\lambda$ is set to 4 is shown in Figure 1 . As a result, it is expected that our new updating mechanism can provide more choices of exploration and exploitation portions as compared to the linear equation. Furthermore, this modification can cover more real applications optimization problems. Finally, the I-GWO will follow the same procedure of GWO by replacing (9) with (10).

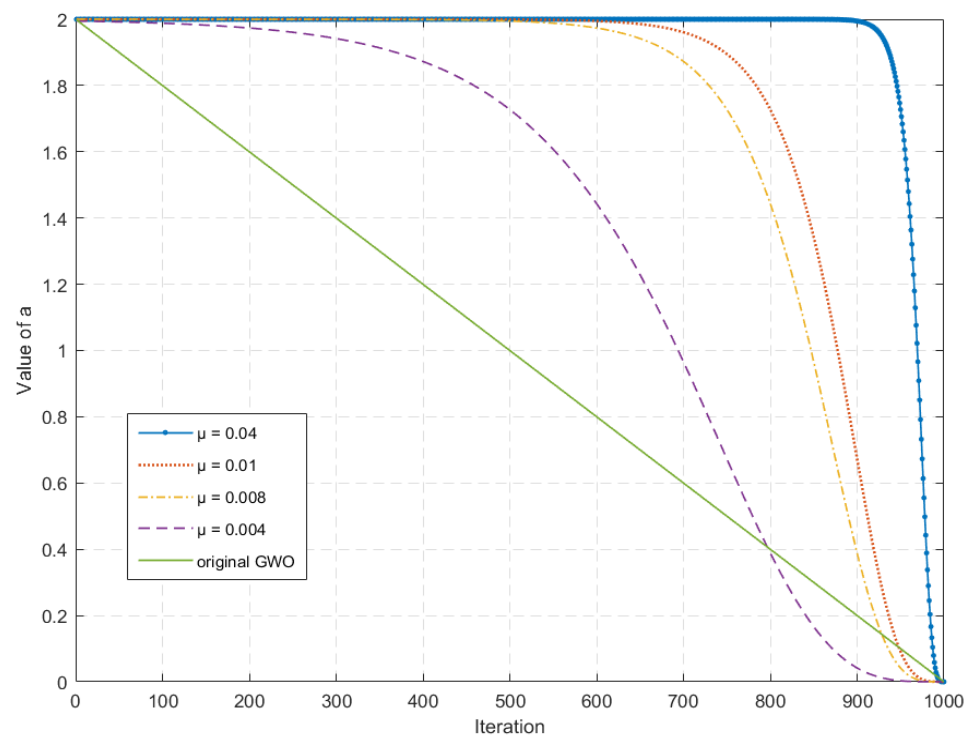

Figure 1. Value of $\tilde{a}$ for different $\mu(\sigma=2, \lambda=4)$

\subsection{Applications of I-GWO for improving energy production of wind farm}

The procedure to apply the proposed I-GWO for improving energy production of wind plant is shown in this section. By applying the I-GWO in the previous section, the procedure for data-driven control of wind plant is given as follows:

Step 1: Select the values of $\sigma, \mu$ and $\lambda$ in (10).

Step 2: Execute the I-GWO by setting $\bar{E}=g_{i}$ and $h_{k}(k=1,2, \ldots, m)=v_{i j}(j=1,2, \ldots, n)$ for each $i$.

Step 3: The algorithm stops with the solution $h_{k}{ }^{*}(k=1,2, \ldots, m)=v_{j}^{\alpha}(j=1,2, \ldots, n)$ after $T$ iterations and the corresponding total energy production $\bar{E}\left(h_{1} *, h_{2} *, \ldots, h_{m} *\right)$ is observed. Note that $v_{j}^{\alpha}$ is $j$ th element of the vector $v^{\alpha}$. 


\section{NUMERICAL RESULTS}

The evaluation of the proposed method is discussed in this section. The first question in this study sought to determine the optimal controller parameters of the turbines so that the total energy production of the wind plant is increased. In this study, the effectiveness of the I-GWO is evaluated on a row of 10 turbines of wind plant, as shown in Figure 2. The wind plant model is adopted for ten turbines $(m=10)$ with $80 \mathrm{~m}$ of turbine rotor diameter on each. The location of the wind turbine is positioned in a row with the same $560 \mathrm{~m}$ distance between each turbine. The values of air density and the turbulence gradient parameters are $\rho=1.225 \mathrm{~kg} / \mathrm{m}^{3}$ and $\varphi=0.04$, respectively. In order to experience large turbulence effect, it is assumed that the wind direction is 90 degrees to the rotor swept area with a fix incoming wind speed at $U=8 \mathrm{~m} / \mathrm{s}$. The simulation works are done with MATLAB 2019a, Microsoft Window 10, 8GB RAM and Intel Core i7-6700 Processor $(3.41 \mathrm{GHz})$.

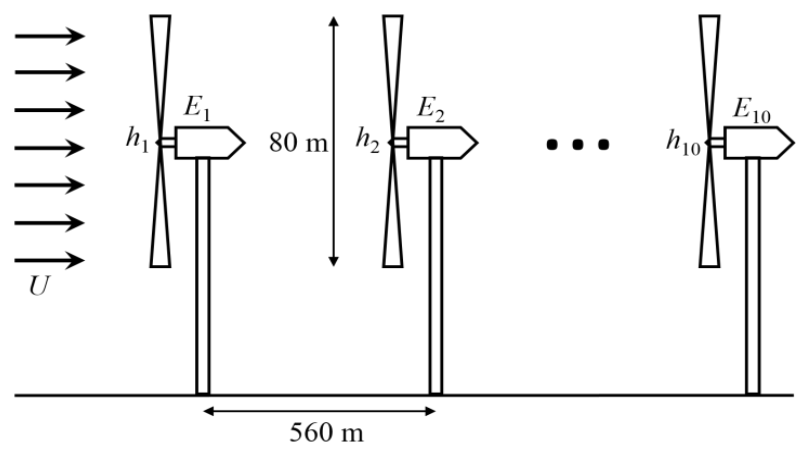

Figure 2. The location of 10 turbines in wind plant

The comparative assessment for I-GWO with original GWO and modified GWO [12] has been done by setting the maximum number of iterations, $T$ to be 1000 and number of agents, $N$ to 10 that contributes to 10,000 number of function evaluations. The coefficients of I-GWO is set as $\mu=0.0005, \lambda=4$ and $\sigma=2$, after performed several initial investigations. For a fair comparison, the $N$ and $T$ for original GWO and modified GWO are set identical with I-GWO. The initial control parameter of each turbine for I-GWO, original GWO and modified GWO are set randomly between ranges of $[0,1 / 3]$. Note that the value of $1 / 3$ is considered as the optimum controller parameter of individual turbine produced by the designer before it is placed in the wind plant. Here, 100 trials are carried out for executing all approaches in order to observe the performance of each method due to the randomization effect.

Table 1 provides the summary statistics for total energy production for the 10,000 number of function evaluations. It can be seen from the data, I-GWO has surpassed original GWO and modified GWO [12] in terms of mean, best, worst and standard deviations of total energy production, after 100 independent trials. The I-GWO yields the highest best total energy production with the value of 4.7648415724 MW, same with modified GWO, followed by the original GWO (4.7648412912 MW). Interestingly, there was also a similar trend for the worst and mean values of the total energy production. The most striking result to emerge from the data is that the standard deviation of I-GWO is lower compared to other methods. This indicates that I-GWO is robust to the stochastic effect while consistently improving total energy production. As shown in Figure 3, the I-GWO convergence curve reported significantly faster compared to the original GWO and modified GWO. Comparing these results, it can be seen that I-GWO has able to balance the exploration and exploitation very well to avoid premature convergence.

On the other hand, based on the obtained optimal controller parameters, the best optimal controller parameters of the I-GWO are recorded as $h_{k}{ }^{*}(k=1,2, \ldots, 10)=(0.2061,0.1611,0.1648,0.1651,0.1698$, $0.1173,0.2258,0.1877,0.1837,0.3333)$. It shows that the optimal controller parameter value of the first turbine is larger compared to the values of middle turbines, but still less than the $1 / 3$. However, the value in the final turbine is maintained as the initial controller parameter, which is $1 / 3$. It shows that the optimum values of the controller parameters of the upstream wind turbines are lower than the $1 / 3$ to reduce the turbulence effect and increase the accumulation of wind speed to the downstream turbines. In contrast, since there is no more downstream turbine for the final turbine, its controller parameter is fixed at the full capacity of $1 / 3$. This trend is similar to existing investigation on data-driven control of wind plant, e.g., [4], while improving the total energy production. 
Generally, statistical results over 100 independent runs do not compare each of the runs because it is still a possible chance that the superiority happens despite its low probability in 100 runs. Therefore, a nonparametric statistical test is performed to compare the results of each run and decide whether the better algorithms is significant or not. In this study, the Wilcoxon rank-sum test is used to determine the significance of the results. Wilcoxon rank sum test stated that the better algorithm should be significant if the p-value less than $5 \%$. Table 2 shows the p-values obtained from the test. From the results, it is shown that the significant superiority of I-GWO compared to original GWO and modified GWO based on the p-values that less than 0.05 . In summary, these results show that the proposed I-GWO has good potential in increasing the total energy production of the wind plant.

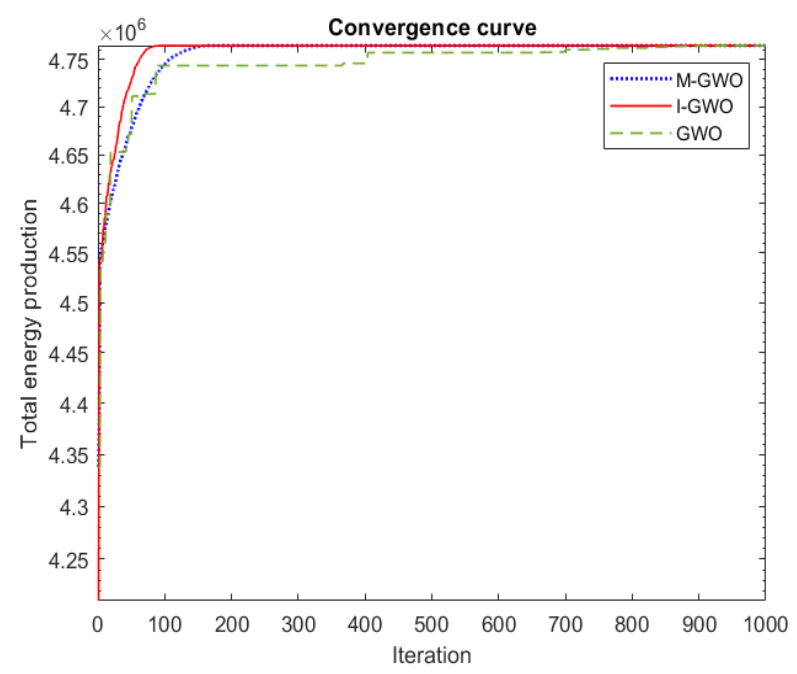

Figure 3. Convergence curve

Table 1. Comparative assessment of total energy production between I-GWO, M-GWO and GWO

\begin{tabular}{cccc}
\hline Statistical results & I-GWO & M-GWO [12] & GWO [12] \\
\hline Mean (MW) & 4.7648415724 & 4.7648415723 & 4.7648390511 \\
Best (MW) & 4.7648415724 & 4.7648415724 & 4.7648412912 \\
Worst (MW) & 4.7648415724 & 4.7648415720 & 4.7648339341 \\
Standard Deviation & $1.6407 \times 10^{-9}$ & $6.678 \times 10^{-5}$ & 1.3615 \\
\hline
\end{tabular}

Table 2. Results from Wilcoxon rank sum test

\begin{tabular}{cc}
\hline Algorithm & p-value \\
\hline I-GWO vs. M-GWO & $3.7369 \mathrm{e}-35$ \\
I-GWO vs. GWO & $3.7369 \mathrm{e}-35$ \\
\hline
\end{tabular}

\section{CONCLUSION}

This study set out to improve the wind plant control parameters for a single row wind plant of 10 turbines by using a new Improved Grey Wolf Optimizer (I-GWO). This study has shown that the proposed I-GWO has produced a slightly higher total energy production compared to the original GWO and previously published modified GWO with more consistent results. In general, therefore, it seems that I-GWO has good potential for data-driven control of wind plant. This research will serve as a base for future studies to investigate the effectiveness of other multi-agent-based optimization for wind plant problem. In terms of directions for future research, further work could be done by implementing I-GWO to solve the problems for other real plant applications.

\section{ACKNOWLEDGEMENTS}

This research is fully funded by the Research Management Centre (RMC), Universiti Tun Hussein Onn Malaysia (UTHM) under the Tier 1 Grant Vot. No. H227. 


\section{REFERENCES}

[1] M. A. Ahmad, S. I. Azuma, and T. Sugie, "A model-free approach for maximizing power production of wind farm using multi-resolution Simultaneous Perturbation Stochastic Approximation," Energies, vol. 7, no. 9, pp. 5624-5646, 2014.

[2] P. M. O. Gebraad et al., "A data-driven model for wind plant power optimization by yaw control, " 2014 American Control Conference, Portland, OR, pp. 3128-3134, 2014.

[3] J. R. Marden, S. D. Ruben, and L. Y. Pao, "Surveying game theoretic approaches for wind farm optimization," in 50th AIAA Aerospace Sciences Meeting Including the New Horizons Forum and Aerospace Exposition, 2012.

[4] J. R. Marden, S. D. Ruben and L. Y. Pao, "A Model-Free Approach to Wind Farm Control Using Game Theoretic Methods," in IEEE Transactions on Control Systems Technology, vol. 21, no. 4, pp. 1207-1214, July 2013.

[5] F. van Dam, P. Gebraad, and J.-W. van Wingerden, "A maximum power point tracking approach for wind farm control," Wiley Online Library, vol. 18, no. 3, pp. 9-11, Feb 2014.

[6] M. R. Hao, M. A. Ahmad, R. M. T. Raja Ismail, and A. N. K. Nasir, "Performance evaluation of random search based methods on model-free wind farm control," in Intelligent Manufacturing \& Mechatronics, Springer, Singapore, pp. 657-670, 2018.

[7] M. R. Hao, R. M. T. R. Ismail and M. A. Ahmad, "Using spiral dynamic algorithm for maximizing power production of wind farm," 2017 International Conference on Applied System Innovation (ICASI), Sapporo, pp. 1706-1709, 2017.

[8] J. Park and K. H. Law, "A data-driven, cooperative wind farm control to maximize the total power production," Applied Energy, vol. 165, pp. 151-165, March 2016.

[9] M. H. Suid, M. Z. M. Tumari, and M. A. Ahmad, "A modified sine cosine algorithm for improving wind plant energy production," Indonesian Journal of Electrical Engineering and Computer Science (IJEECS), vol. 16, no. 1, pp. 101-106, Oct 2019.

[10] M. A. bin M. Idris, M. R. Hao, and M. A. Ahmad, "A data driven approach to wind plant control using moth-flame optimization (MFO) algorithm," International Journal on Advanced Science Engineering Information Technology, vol. 9, no. 1, pp. 18-23, 2019.

[11] S. Mirjalili, S. M. Mirjalili, and A. Lewis, "Grey Wolf Optimizer," Advances in Engineering Software, vol. 69, pp. 46-61, March 2014.

[12] M. Z. M. Tumari, M. H. Suid, and M. A. Ahmad, "A modified grey wolf optimizer for improving wind plant energy production," Indonesian Journal of Electrical Engineering and Computer Science (IJEECS), vol. 18, no. 3, pp. 1123-1129, June 2020.

[13] M. H. Sulaiman, Z. Mustaffa, M. R. Mohamed, and O. Aliman, "Using the gray wolf optimizer for solving optimal reactive power dispatch problem," Applied Soft Computing, vol. 32, pp. 286-292, July 2015.

[14] E. Gupta and A. Saxena, "Grey wolf optimizer based regulator design for automatic generation control of interconnected power system," Cogent Engineering, vol. 3, no. 1, pp. 1-20, 2016.

[15] S. Mohanty, B. Subudhi and P. K. Ray, "A New MPPT Design Using Grey Wolf Optimization Technique for Photovoltaic System Under Partial Shading Conditions," in IEEE Transactions on Sustainable Energy, vol. 7, no. 1, pp. 181-188, Jan 2016.

[16] S. Mohanty, B. Subudhi and P. K. Ray, "A Grey Wolf-Assisted Perturb \& Observe MPPT Algorithm for a PV System," in IEEE Transactions on Energy Conversion, vol. 32, no. 1, pp. 340-347, March 2017.

[17] B. S. Yildiz and A. R. Yildiz, "Comparison of grey Wolf, whale, water cycle, ant lion and sine-cosine algorithms for the optimization of a vehicle engine connecting rod," Material Testing, vol. 60, no. 3, pp. 311-315, 2018.

[18] M. Radmanesh, M. Kumar, and M. Sarim, "Grey wolf optimization based sense and avoid algorithm in a Bayesian framework for multiple UAV path planning in an uncertain environment," Aerospace Science and Technology, vol. 77, pp. 168-179, June 2018.

[19] S. S. Rajput, V. K. Bohat, and K. V. Arya, "Grey wolf optimization algorithm for facial image super-resolution," Applied Intelligent, vol. 49, no. 4, pp. 1324-1338, 2019.

[20] L. Li, L. Sun, J. Guo, J. Qi, B. Xu, and S. Li, "Modified Discrete Grey Wolf Optimizer Algorithm for Multilevel Image Thresholding," Computational Intelligence and Neuroscience, vol. 2017, article id 3295769, pp. 1-16, Jan 2017.

[21] M. H. Qais, H. M. Hasanien and S. Alghuwainem, "A Grey Wolf Optimizer for Optimum Parameters of Multiple PI Controllers of a Grid-Connected PMSG Driven by Variable Speed Wind Turbine," in IEEE Access, vol. 6, pp. 44120-44128, 2018.

[22] S. Kapoor, I. Zeya, C. Singhal, and S. J. Nanda, “A Grey Wolf Optimizer Based Automatic Clustering Algorithm for Satellite Image Segmentation,” in Procedia Computer Science, vol. 115, pp. 415-422, 2017.

[23] A. Yahiaoui, F. Fodhil, K. Benmansour, M. Tadjine, and N. Cheggaga, "Grey wolf optimizer for optimal design of hybrid renewable energy system PV-Diesel Generator-Battery: Application to the case of Djanet city of Algeria," Solar Energy, vol. 158, pp. 941-951, Dec 2017.

[24] M. A. Ahmad, Z. Musa, M. H. S. Suid, and M. Z. M. Tumari, "Grey Wolf Optimizer for Identification of Liquid Slosh Behavior using Continuous-Time Hammerstein Model," Bulletin of Engineering and Informatics (BEEI), vol. 9, no. 2, pp. 542-549, April 2020.

[25] J. D. Grunnet, M. Soltani, T. Knudsen, M. Kragelund, and T. Bak, "Aeolus toolbox for dynamics wind farm model, simulation and control," in European Wind Energy Conference and Exhibition 2010, EWEC 2010, pp. 1-10, 2010. 


\section{BIOGRAPHIES OF AUTHORS}
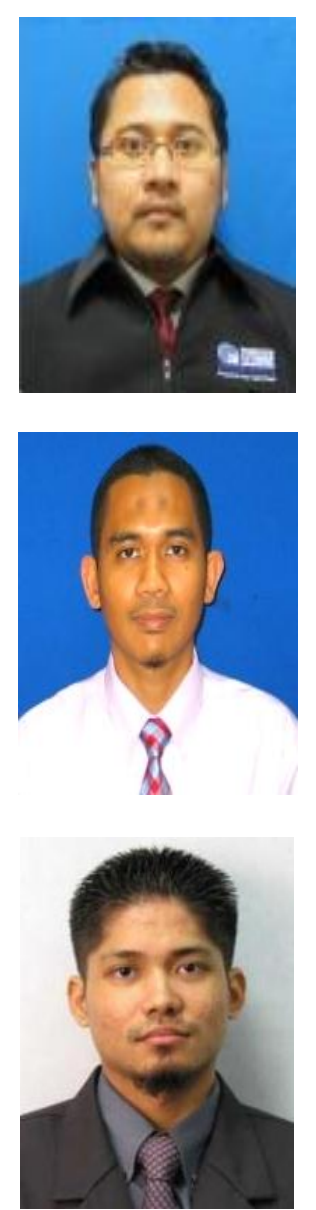

Mohd Zaidi Mohd Tumari is received his first degree in B.Eng. Electrical Mechatronics in 2008 from University of Technology Malaysia (UTM) in Johor, Malaysia and in 2010, he received a Master's degree in M.Eng. Mechatronics and Automatic Control from University of Technology Malaysia (UTM). He has an experience as a research assistant at Robotic Laboratory in University of Technology Malaysia (UTM) in 2008, Tutor in Universiti Pendidikan Sultan Idris (2008-2011) and Lecturer in Universiti Malaysia Pahang (2011-2014). As an academician and researcher, he has involved in many research and development in field of mechatronics, control and robotics. Currently, he is a Lecturer in Faculty of Engineering Technology, Universiti Teknikal Malaysia Melaka (UTeM). His current research interests are vibration and sway control, input shaping, crane system, liquid slosh system and flexible robot manipulator.

Mohd Muzaffar Zahar received his B. Eng. in Electrical Engineering (Mechatronics) with honours from Universiti Teknologi Malaysia in 2008. He also graduated in same university with a M. Eng. (Electrical Engineering) in 2013. Begin in education system since 2010 at Industrial Training Institute of Marang, then at Advanced Training Centre (ADTEC) of Melaka, next in National Youth High Skill Training Institutes of Pagoh and now as lecturer in Universiti Tun Hussein Onn Malaysia (UTHM). Based on his experienced in different institution, the research interest includes routing in wireless sensor network, industrial automation and application of control engineering.

Mohd Ashraf Ahmad received his first degree in B.Eng. Electrical Mechatronics in 2006 from University of Technology Malaysia (UTM) in Johor, Malaysia. In 2008, he received a Master degree in M.Eng. Mechatronics and Automatic Control from University of Technology Malaysia (UTM). He is a lecturer in Faculty of Electrical and Electronics Engineering, Universiti Malaysia Pahang (UMP). He also became a professional member in IEEE, SICE, ACA and IAENG. His current research interests are model-free control, stochastic approximation, vibration and sway control, input shaping, crane system and flexible robot manipulator. 